\title{
Measurement of current taken by fluorescent lights in the frequency range $2-150 \mathrm{kHz}$
}

\author{
E. O. A. Larsson, C. M. Lundmark and M. H. J. Bollen, Fellow, IEEE
}

\begin{abstract}
This paper discusses measurements taken on fluorescent lights in the frequency range between 2 and $150 \mathrm{kHz}$. The presentation of the measurement results form an important part of the paper. The measurement results are presented in time-domain, in the frequency domain and in the time-frequency domain (by means of a "spectrogram"). The latter allows the study of sub-cycle changes in the current spectrum. The current taken by one lamp is compared with the current taken by two, three and four lamps. The relative high-frequency distortion reduced for frequencies above $15 \mathrm{kHz}$.

Index Terms--Power quality, Electromagnetic compatibility (EMC), Short-time Fourier transform, energy-efficient lighting.
\end{abstract}

\section{INTRODUCTION}

$\mathrm{M}$ ost of the lights today that are in uses in offices, schools, hospitals and other public buildings are fluorescent lamps powered by high frequency ballast. The benefits of this type of technology includes the reduction of power losses and flicker, and a longer life time compared with traditionally fluorescent lamps power by magnetic ballasts [1], [2].

Fluorescent lamps with high-frequency ballast also show lower current distortion for those harmonic components that are considered in existing standards, i.e. up to $2 \mathrm{kHz}$ [7],[8],[9],[10]. The high-frequency ballast converts ac to dc voltage and contains a capacitive filter bank. The filter is needed to limit both low-frequency distortion and highfrequency distortion. Most high frequency ballasts are designed with active power factor correction (APFC) that reduces the low-frequency distortion to comply with harmonic standards, in particular IEC 61000-3-2 [4].

The reduction in low-frequency distortion is however associated with an increase in high-frequency distortion. The consequences of this high-frequency distortion remain an uncharted territory, but there are sufficient indications of potential interference to justify further study of the various phenomena [11].

In this paper, the current taken by a fluorescent lamp is measured and analysed as an example of a high-frequency disturbance. For the purpose of this paper, "high-frequency" is synonym with the frequency range from 2 to $150 \mathrm{kHz}$. The measurement set-up is presented in Section II; various ways

Financial support from Skellefteå Kraft Elnät is gratefully acknowledged.

The authors are with EMC-on-Site, Luleå University of Technology, 93187 , Skellefteå, Sweden (e-mail: anders.1.larsson@ltu.se). M.H.J. Bollen is also with STRI AB, 771 80, Ludvika, Sweden. of presenting the results in Section III; the change in current spectrum for multiple lamps in Section IV; and conclusions in Section V.

\section{MeASUREMENT SETUP}

A set of measurements on modern fluorescent lamps in an office at the university has been carried out. The aim of the measurements was to map the high-frequency contents of the current taken by such lamps. Each lamp contains two electronic ballasts powering three fluorescent tubes of $28 \mathrm{~W}$ each. The ballasts are designed for nominal voltages between 220 and $240 \mathrm{~V}$ and have a power factor equal to 0.98 .

A Hioki 8855 power-quality monitor was used for the measurements. Pearson, model 411, current monitors were used with the low frequency $3 \mathrm{~dB}$ point at $5 \mathrm{~Hz}$ and high frequency $3 \mathrm{~dB}$ point at $20 \mathrm{MHz}$. The currents to the fluorescent lamps were obtained with a sampling frequency of $1 \mathrm{MS} / \mathrm{s}$ over a $200 \mathrm{~ms}$ window resulting in 200001 data points. Apart from the anti-aliasing filter, no additional filtering has been performed on the currents. The waveform used for the analysis therefore contains power-system frequency components, low-frequency harmonics and highfrequency harmonics, in their original ratio.

The main interest of the measurement was the frequency range between 2 and $150 \mathrm{kHz}$, but the nature of the measurements, in particular the absence of a high-pass filter, made that we were also able to draw conclusions about the frequency range below $2 \mathrm{kHz}$.

Measurements were performed at different times of the day, in one case every 10 minutes during a 24-hour period. No large changes in spectrum were found, with the exception of periods during which power-line communication was taking place.

During some of the measurements, the voltage waveform was recorded together with the current waveform. An analogue high-pass filter was used to reduce the power-system frequency component. This was needed, as the dynamic range of the instrument was not large enough to give sufficient resolution to detect high-frequency voltage components.

\section{Presentation of MeAsurement Results}

\section{A. Time-domain waveform}

Fig. 1 shows measurement in time domain taken on one lamp. The rms current drawn by the lamp is about $0.43 \mathrm{~A}$ and 
the time measurement reveals that the current drawn by the lamp is nearly sinusoidal. There are only some minor defects near the zero-crossing of the current and some small defects at both the positive and the negative peak of the sinusoidal wave form.

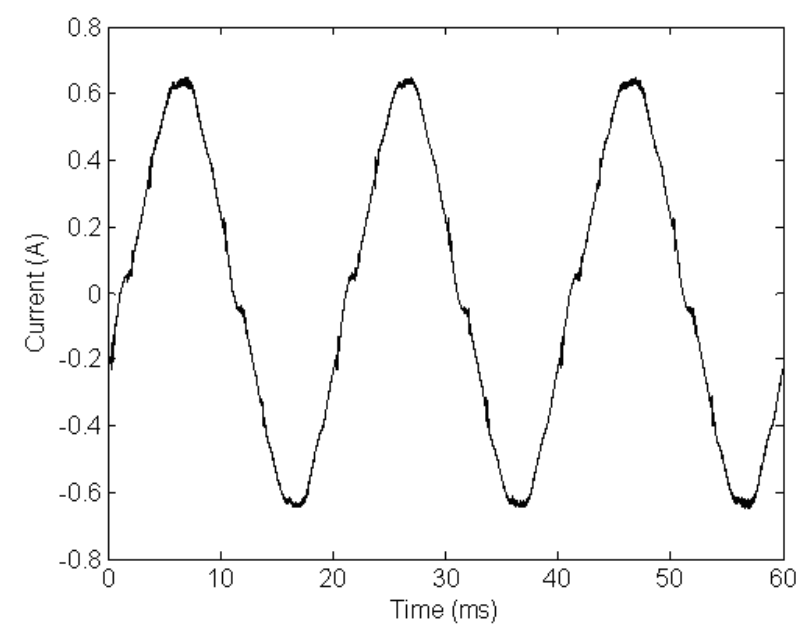

Fig. 1. The current drawn by one fluorescent lamp during three cycles of the power-system frequency.

The current signal as shown in Fig. 1 is dominated by the power-system frequency component at $50 \mathrm{~Hz}$. However a closer look at the signal reveals the presence of highfrequency components. Fig. 2 shows three 1-ms windows, one at the peak, one at the zero-crossing and one somewhere in between. The latter window shows a short-duration damped oscillation, somewhat similar to "notching" as observed with some large motor drives. The 1-ms zoom of the waveform reveals that the high-frequency components vary during one cycle. A further look on the time plot discloses that the appearance of high-frequency components is repetitive. Note that the lamp contains a filter with a capacitive input in gridside. A high-frequency disturbance in the voltage will cause a current through this filter.
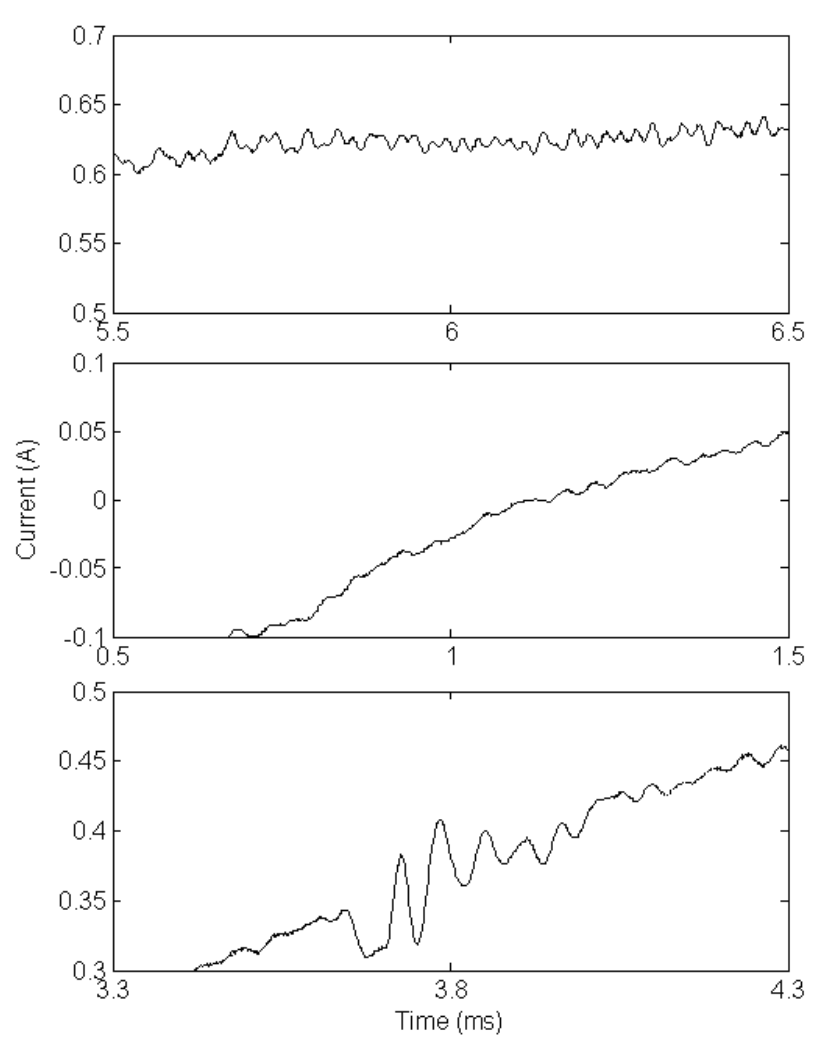

Fig. 2. High frequency content in the current drawn by one fluorescent lamp at maximum (top), at zero-crossing (middle) and around a "notch" (bottom).

\section{B. The frequency spectrum, up to $2 \mathrm{kHz}$.}

The spectrum of the current has been obtained by applying a discrete Fourier transform (DFT) to the 200-ms window. The resulting components (at $5-\mathrm{Hz}$ intervals) have been merged into harmonic groups as prescribed in IEC 61000-4-7 [5]. The resulting spectrum for one lamp is shown in Fig. 3 as a percentage of the power-system component. As mentioned before is the lamp drawing almost sinusoidal current from the utility network and the harmonic current is less than $8 \%$ of frequency up to the $40^{\text {th }}$ harmonic. The third harmonic has the highest amplitude approx. $7.5 \%$ of the fundamental and the fifth harmonic is less than $2 \%$. 


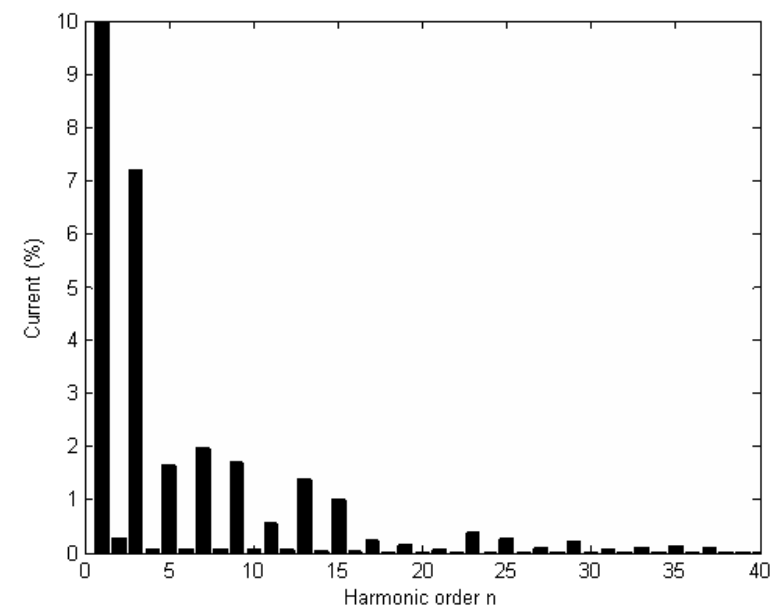

Fig. 3. Low-frequency harmonic content in percentage of the input current at fundamental drawn by one HF-fluorescent lamp.

IEC 61000-3-2 [4] regulates the harmonic current emission from equipment up to $16 \mathrm{~A}$ per phase. The standard divides equipments into four classes, class $\mathrm{A}$ to $\mathrm{D}$. Class $\mathrm{C}$ regulates lightning equipment and HF-fluorescent light is regulated by current limits in Table I. The highest individual harmonic limits apply to third harmonic and as mentioned before, the lamp has a power factor $(\lambda)$ at 0.98 , which gives a limit of $29.4 \%$ third harmonic. Comparing Fig. 3 with Table I shows that the lamp fully complies with the standard.

TABLE I

IEC 61000-3-2, LIMITS FOR CLASS C EQUIPMENT

\begin{tabular}{|l|l|}
\hline Harmonic order & $\begin{array}{c}\text { Maximum permissible harmonic current } \\
\text { expressed as a percentage of the input current at } \\
\text { fundamental frequency in \% }\end{array}$ \\
\hline 2 & 2 \\
3 & $30 \cdot \lambda^{*}$ \\
5 & 10 \\
7 & 7 \\
9 & 5 \\
$11 \leq \lambda \leq 39$ & 3 \\
(odd harmonics only) & \\
\hline & \\
\hline
\end{tabular}

\section{The frequency spectrum $2-150 \mathrm{kHz}$}

The above-mentioned spectrum with $5-\mathrm{Hz}$ resolution extends far beyond the 40th harmonic ( $2 \mathrm{kHz}$ ). Fig. 4 shows the high frequency contents of the current to one lamp in the range from 2 to $150 \mathrm{kHz}$. The amplitude of the highfrequency components varies over a factor of 100. The continuous noise level above $80 \mathrm{kHz}$ is due to the limitations of the measurement system. A simulation of an ideal sinusoidal waveform quantified with the same resolution as used in the measurement system used, confirmed that the continuous noise level starting at about $80 \mathrm{kHz}$ is due to the quantization error made in the A/D converter. Filtering of the $50-\mathrm{Hz}$ component is needed to reduce this quantization noise.

The lower-frequency components dominate the spectrum, with broadband peaks around 20 and $45 \mathrm{kHz}$. Note the difference in shape between these two peaks. A narrowband peak is visible at $119.8 \mathrm{kHz}$. This signal was not visible at earlier measurements, so it is reasonable to assume that the signal origin is found elsewhere in the system.

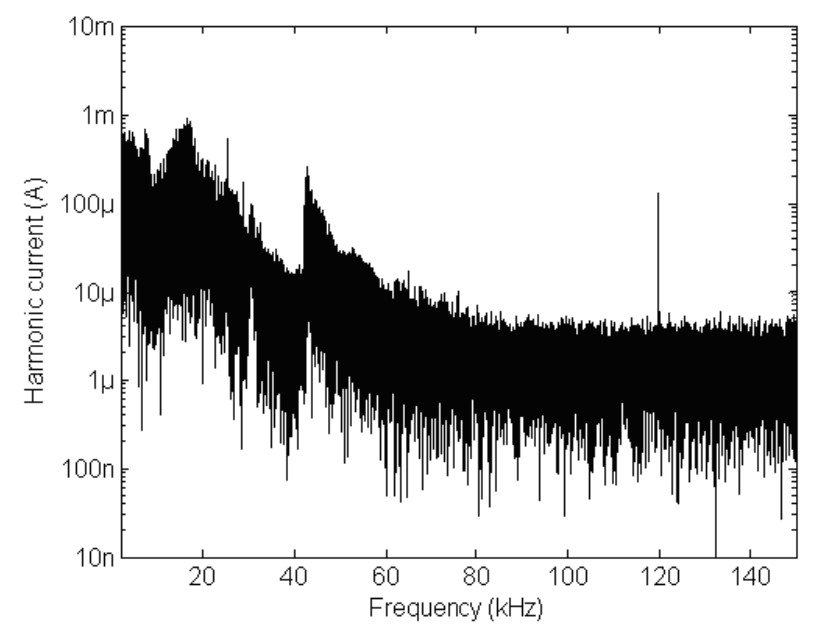

Fig. 4. High-frequency contents in the current drawn by the one fluorescent lamp; frequency components with 5- $\mathrm{Hz}$ resolution.

Standard document IEC 61000-4-7 [5] gives in an informative annex a method for characterizing measurements between 2 to $9 \mathrm{kHz}$. The main differences between the methodologies for low-frequency harmonic and these higherfrequency measurements are that the sampling window is 100 ms (approximately 5 cycles for $50 \mathrm{~Hz}$ and 6 cycles for $60 \mathrm{~Hz}$ ) and that there is no need to synchronize the sampling to the power-system frequency. The spectrum is obtained by applying a DFT with a rectangular window to the basic measurement window of $100 \mathrm{~ms}$. This results in a spectrum with $10-\mathrm{Hz}$ separation between the frequency components. The output of the DFT is grouped into frequency bands (groups) with bandwidth $200 \mathrm{~Hz}$. The frequency band 2000$2200 \mathrm{~Hz}$ is referred to as the $2100-\mathrm{Hz}$ group, etc. Each group thus contains 20 frequency components. The magnitude of the distortion in frequency band (group) $b$ is obtained from taking the root-square value over all frequency components $C_{f}$ with $f$ between $b-90 \mathrm{~Hz}$ and $b+100 \mathrm{~Hz}$ as in (1). The bandwidth has been chosen to be in accordance with CISPR 16-1.

$$
G_{b}=\sqrt{\sum_{b-90 \mathrm{~Hz}}^{b+100 \mathrm{~Hz}} C_{f}^{2}}
$$

For the measurements presented in this paper, a 200-ms window has been used, resulting in a frequency resolution of $5 \mathrm{~Hz}$. The $200-\mathrm{Hz}$ values have been obtained by taking the root mean square over 40 frequency values, instead of 20 according to (1). Whereas IEC 61000-4-7 only refers to frequencies up to $9 \mathrm{kHz}$, the same grouping has been applied up to $150 \mathrm{kHz}$. The spectrum shown in Fig. 5 covers the same frequency band as Fig. 4 but the signals are grouped in 200 $\mathrm{Hz}$ bands. The grouping and smoothing makes the current less noisy and individual characteristics are easier to detect. It becomes visible, for example, that two narrow-band components are present around $30 \mathrm{kHz}$. 


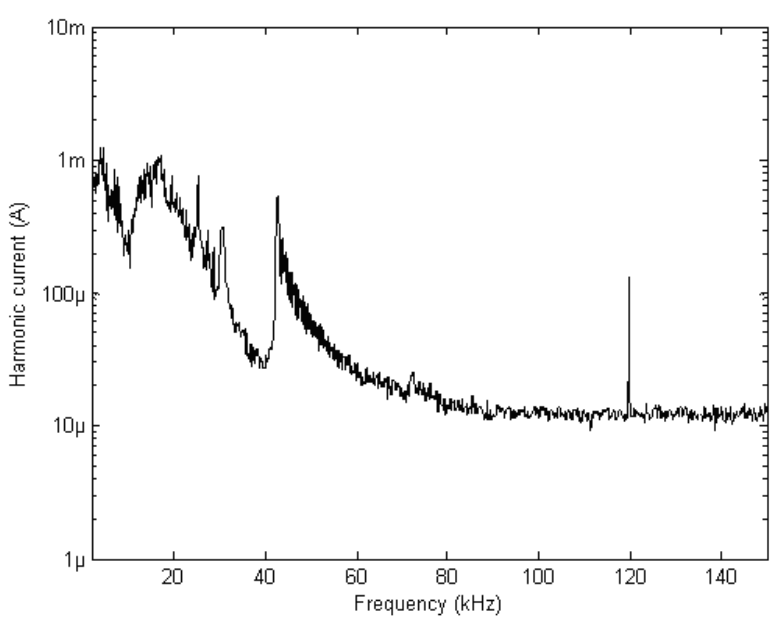

Fig. 5. High-frequency contents in the current drawn by one fluorescent lamp, frequency components grouped into 200-Hz bands according to IEC 61000-4-7.

\section{Time-frequency domain}

When looking at the current signal in time domain it was seen that the character of the high-frequency component varies throughout one cycle of the power-system frequency. Successive cycles are identical but within one cycle the signal is non-stationary. When applying the DFT tool it is always explicitly assumed that the signal is stationary.

A method to analyse sub-cycle changes in spectral contents is by using the short-time Fourier transform (STFT) [6]. Time and frequency axes are each split into equal intervals or windows, resulting in time-frequency bins. For each bin a signal magnitude or intensity is calculated by applying a DFT to the time interval. The result may be presented as a spectrogram where different colours or greyscales correspond to different signal intensities.

Note that time and frequency resolution cannot be independently chosen. A higher time resolution corresponds with a lower frequency resolution; their product being constant. The spectrogram for the current taken by one lamp (Fig.1) is shown in Fig. 6 where the time step is $1 \mathrm{~ms}$ and the frequency step $1 \mathrm{kHz}$. The spectrogram was calculated by using the "spectrogram" function in Matlab. The DFT was applied to 1-ms Hanning windows and updated every $0.1 \mathrm{~ms}$. The separation between frequency values is $1 \mathrm{kHz}$.

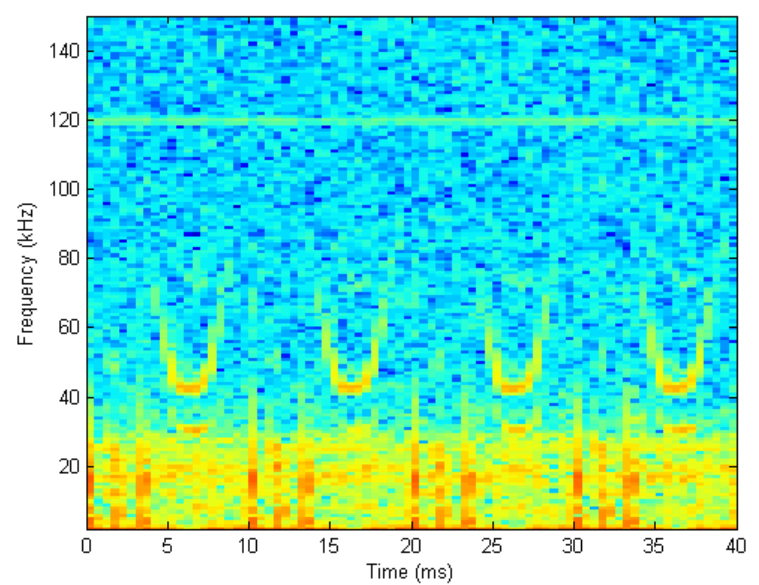

Fig. 6. STFT spectrogram for the current taken by one lamp.

The spectrogram shows a number of "time-frequency components":

- A continuous stationary component at $120 \mathrm{kHz}$. This component corresponds to the narrow peak in Fig. 5. The reduction in amplitude for increasing number of lamps (Fig. 10) points to an origin in the lamps.

- An intermittent variable frequency component with lowest frequency and highest intensity around current maximum and minimum. Such a component is expected when hysteresis control is used. This component only appears in the voltage when the lamps are turned on. This component corresponds to the triangular-shaped peak around $45 \mathrm{kHz}$ in Fig. 5 .

- A number of components, narrow in time, broad in frequency. These components occur four times per cycle, before and after the current zero-crossings. The components are present in the voltage already before the lamps are switched on. Their origin is unknown, but an external origin is most likely.

\section{CURREnT TAKEN By MULTIPLE LAMPS}

\section{A. Multiple lamps, up to $2 \mathrm{kHz}$}

The measurements and calculations resulting in Fig. 3 have been repeated for the sum of the currents of two, three and four individual lamps. A comparison for the dominating harmonics is shown in Table II. It is easy to conclude that the spectrum is independent of the number of lamps. There is no cancellation of low-frequency harmonics. This is however not really a concern as the current distortion of other equipment (like computers, TV screens, older-generation fluorescent lamps) is significantly higher, with third-harmonic levels up to $80 \%$.

TABLE II

RELATIVE HARMONIC CURRENT DISTORTION IN PERCENTAGE OF THE FUNDAMENTAL AS A FUNCTION OF THE NUMBER OF LAMPS

\begin{tabular}{|l|l|l|l|l|l|l|l|}
\hline & 3 th & 5 th & 7th & 9th & 11th & 13th & 15th \\
\hline 1 & 7.2 & 1.7 & 1.9 & 1.7 & 0.6 & 1.3 & 1.0 \\
\hline 2 & 7.7 & 1.7 & 2.1 & 1.7 & 0.4 & 1.3 & 0.9 \\
\hline 3 & 7.7 & 1.8 & 1.9 & 1.7 & 0.5 & 1.3 & 1.0 \\
\hline 4 & 7.6 & 1.8 & 2.0 & 1.7 & 0.5 & 1.4 & 1.0 \\
\hline
\end{tabular}




\section{B. Multiple lamps, $2-150 \mathrm{kHz}$}

The calculations resulting in Fig. 5 have been repeated for the current taken by two, three and four identical lamps. A comparison is made for different parts of the spectrum in Fig. 7 through Fig.10. In all cases the harmonic current is given as a percentage of the rms current. The lower part of the spectrum is shown in Fig. 7. The spectra for the four cases are very similar. Like with low-frequency harmonics (see Table II) the relative distortion is very similar for one, two, three or four lamps. There is thus no cancellation between different lamps.

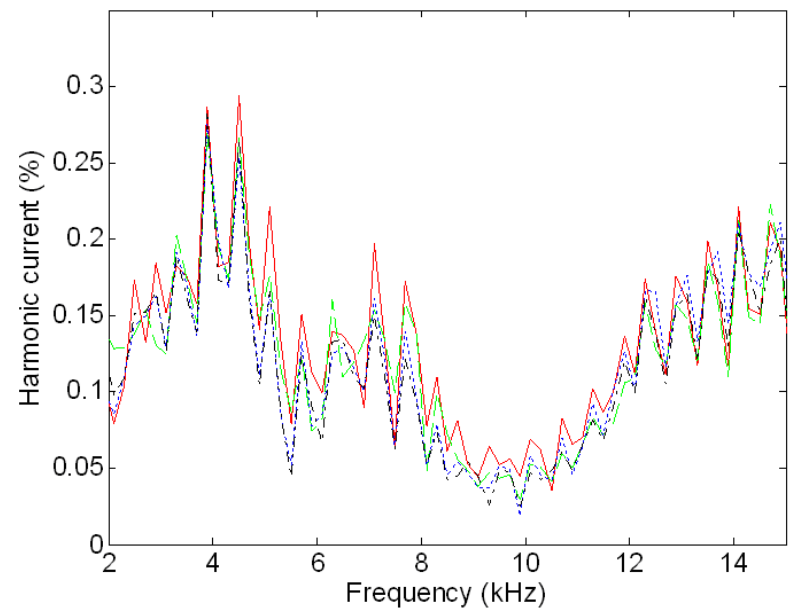

Fig. 7. Spectra of the current in the frequency band $2-15 \mathrm{kHz}$ for one lamp (red solid), two lamps (green dashed), three lamps (blue dotted) and four lamps (black, dash-dot).

Some cancellation between the lamps takes place in the frequency band $15-40 \mathrm{kHz}$, as shown in Fig. 8. The relative distortion generally decreases with increasing number of lamps. However at some frequencies the relative distortion is higher for two lamps than for one lamp.

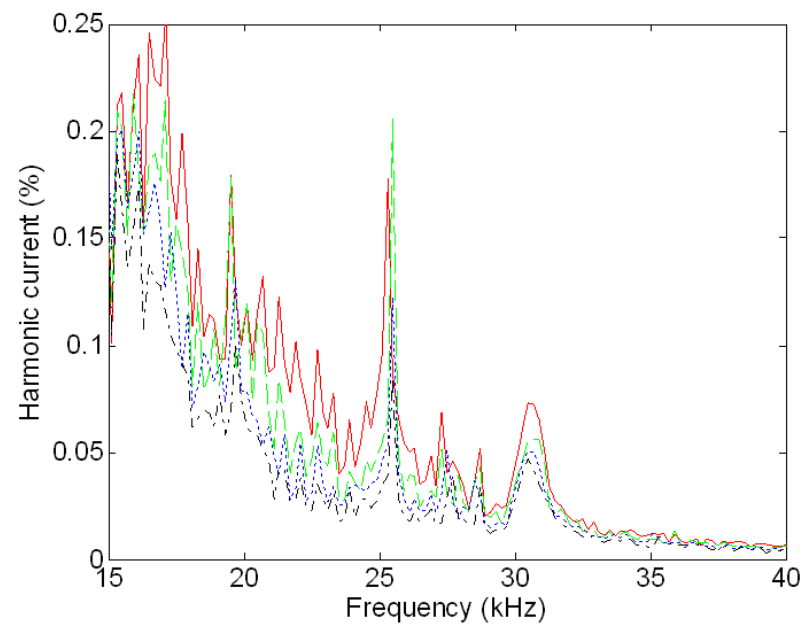

Fig. 8. Spectra of the current in the frequency band $15-40 \mathrm{kHz}$ for one lamp (red solid), two lamps (green dashed), three lamps (blue dotted) and four lamps (black, dash-dot).
The reduction in relative harmonic contents becomes most obvious around 45 kHz (Fig. 9) and around $120 \mathrm{kHz}$ (Fig. 10). The peak at $42 \mathrm{kHz}$ is only one quarter in amplitude for four lamps compared to two lamps. At $119.8 \mathrm{kHz}$ this is a factor two. As mentioned earlier, this $120-\mathrm{kHz}$ signal is not present all the time, so that an external origin appears most reasonable. This would be somewhat in contradiction with the observed reduction of the relative distortion with increasing number of lamps. A possible explanation is that the input impedance of the lamps at this frequency is low compared to the source impedance. The connection of multiple lamps will reduce the voltage distortion and thus the relative current distortion.

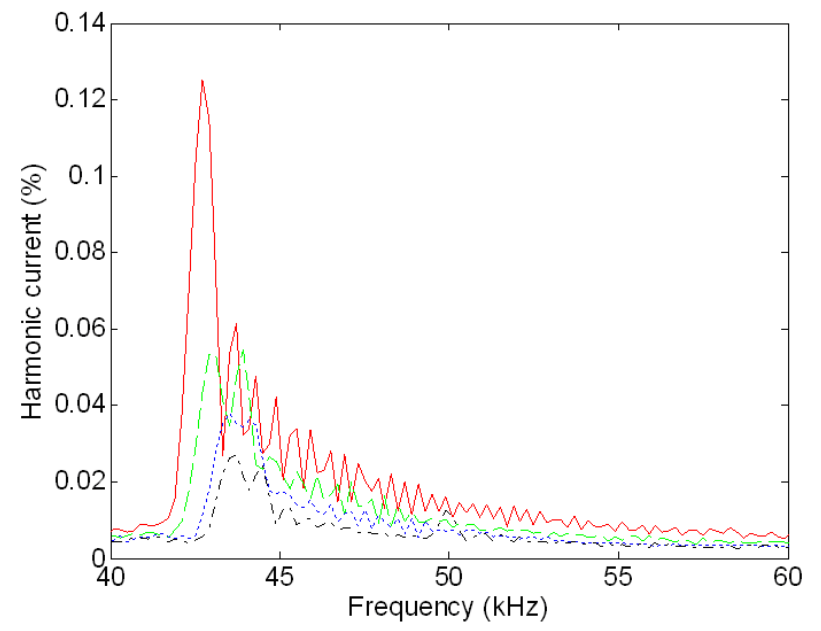

Fig. 9. Spectra of the current in the frequency band $40-60 \mathrm{kHz}$ for one lamp (red solid), two lamps (green dashed), three lamps (blue dotted) and four lamps (black, dash-dot).

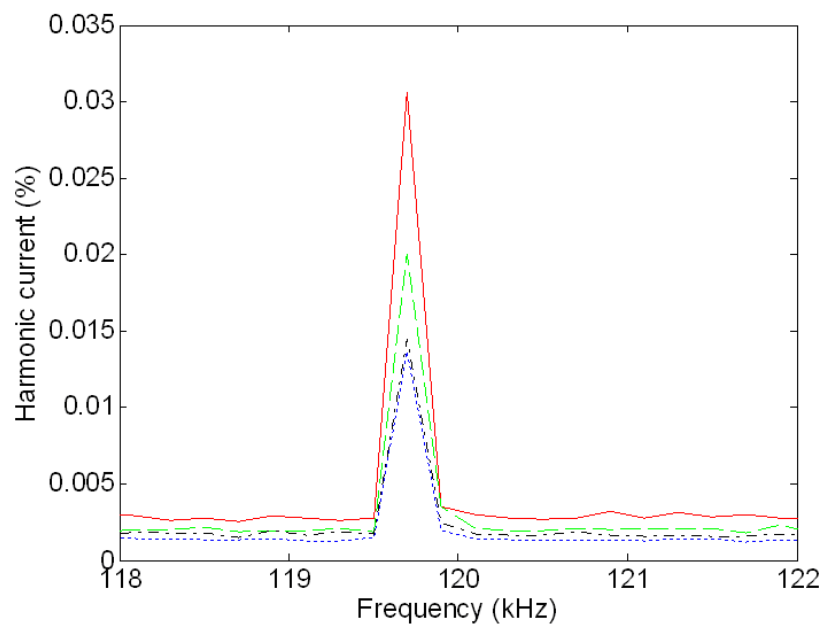

Fig. 10. Spectra of the current around $120 \mathrm{kHz}$ for one lamp (red solid), two lamps (green dashed), three lamps (blue dotted) and four lamps (black, dashdot). 


\section{CONCLUSION}

The current taken by a fluorescent lamp with HF ballast contains frequency components throughout the frequency range $2-150 \mathrm{kHz}$. Some components are stationary (in this case for example at $119.8 \mathrm{kHz}$ ) whereas others show a variation in spectral contents within one cycle of the powersystem frequency. The short-time Fourier transform, in this paper presented in the form of a spectrogram, appears to be a suitable tool to analyse such components.

Comparing the spectra for one, two, three and four lamps shows that at most only minor cancellation occurs up to 15 $\mathrm{kHz}$. For higher frequencies the relative harmonic contents decreases with increasing number of lamps.

Future work needed, includes measurements on more devices, voltage measurements at a number of locations, further development of time-frequency-methods to characterize high-frequency disturbances, component models explaining and reproducing the measured disturbances, and methods to find the origin of the various disturbance components.

\section{REFERENCES}

[1] W. Xiaoming, Z. Ke, Z. Ying, and S. Wenxiang, "Study on Fluorescent Lamp Illumination and Flicker" in Proc. The Fifth International Conference on Power Electronics and Drive Systems PEDS 2003 Vol. 2. pp. 1529 - 1532.

[2] Y. Ji, R. Davis, C. O'Rourke, and E. W. M. Chui, "Compatibility Testing of Fluorescent Lamp and Ballast System," IEEE Trans. on industry applications, vol. 35, pp. 1271-1276, Nov/Dec. 1999.

[3] M. Bollen, and I. GU, Signal Processing of Power Quality Disturbances, New York: Wiley/ IEEE Press, in production.

[4] Limits for harmonic current emissions (equipment input current $\leq 16 \mathrm{~A}$ per phase), IEC 61000-3-2

[5] Testing and measurement techniques- General guide on harmonics and interharmonics measurements and instrumentation, for power supply systems and equipment connected thereto, IEC 61000-4-7:2002.

[6] Y.H. Gu, M.H.J. Bollen, Time-frequency and time-scale domain analysis of voltage disturbances, IEEE Transactions on Power Delivery, vol.15, no.4; Oct. 2000; p.1279-84.

[7] M. Etezadi-Amoli, T. Florence, Power factor and harmonic distortion of energy efficient lamps, IEEE Transactions on Power Delivery, Vol.4, no.3, July 1989 , pp.1965-1969.

[8] I.F. Gonos, M.B. Kostic, F.V. Topalis, Harmonic distortion in electric power systems introduced by compact fluorescent lamps, IEEE Budapest Power Tech, 1999.

[9] R.R. Verderber, O.C. Morse, W.R. Alling, Harmonics from compact fluorescent lamps, IEEE Transactions on Industry Applications, Vol.29, no.3 (May/June 1993), pp.670-674.

[10] M. Waller, Harmonics. Howard Sams, Indianapolis, IN 46214-2041, 1994.

[11] C.M. Lundmark, E.O.A. Larsson, M.H.J. Bollen, Unintended consequences of limiting high-frequency emission by small end-user equipment, IEEE Power Engineering Society, General Meeting 2006 (this conference).

\section{BIOGRAPHIES}

Anders Larsson received the BSc degree from Luleå University of Technology, Skellefteå, Sweden in 1999. Currently he is PhD student at the university. Before starting as a PhD student in 2004 he has worked at Luleå University of Technology as research engineer with power quality and EMC issues. His research interest covers a wide range of electrical power issues and his special emphasis is on power quality and EMC.

Martin Lundmark received the MSc degree from the Royal Institute of Technology, Stockholm, Sweden, Currently he is project manager at EMC on SITE at Luleå University of Technology, Skellefteå, Sweden. Before joining Luleå University of Technology in 1992 as a lecturer he was research engineer at ABB Corporate Research in Västerås. His research interests cover the wide range including electric power system and telecommunication with special emphasis on EMC.

Math Bollen received the MSc and PhD degree from Eindhoven University of Technology, Eindhoven, The Netherlands. Currently he is professor at EMC-onSite, Luleå University of Technology, Skellefteå, Sweden and co-ordinator power quality and EMC at STRI AB, Ludvika, Sweden. Before joining STRI in 2003 he was research associate at Eindhoven University of Technology, Lecturer at University of Manchester Institute of Science and Technology, Manchester, UK and professor in electric power systems at Chalmers University of Technology, Gothenburg, Sweden. His research interests cover a wide range of electric power system issues with special emphasis on power quality and reliability. 